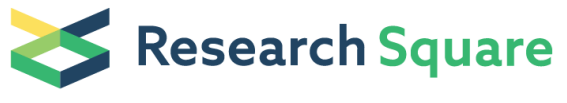 \\ Preprints are preliminary reports that have not undergone peer review. \\ They should not be considered conclusive, used to inform clinical practice, or referenced by the media as validated information.
}

\section{A novel and simple risk model predicts the prognosis for patients with paraquat poisoning}

\section{Yanxia Gao}

Zhengzhou University First Affiliated Hospital

Liwen Liu

Zhengzhou University First Affiliated Hospital

Tiegang Li

Shengjing Hospital of China Medical University

Ding Yuan

Zhengzhou University First Affiliated Hospital

Yibo Wang

Zhengzhou University First Affiliated Hospital

\section{Zhigao Xu}

Zhengzhou University First Affiliated Hospital

\section{Guangying Cui}

Zhengzhou University First Affiliated Hospital

\section{Linlin Hou}

Zhengzhou University First Affiliated Hospital

\section{Yan Zhang}

Zhengzhou University First Affiliated Hospital

\section{Guoyu Duan}

Zhengzhou University First Affiliated Hospital

\section{Changhua Sun}

Zhengzhou University First Affiliated Hospital

\section{Heqi Zhou}

Zhengzhou University First Affiliated Hospital

Lu Che

Zhengzhou University First Affiliated Hospital

\section{Sujuan Li}

Zhengzhou University First Affiliated Hospital

Yi Li

Chinese Academy of Medical Sciences and Peking Union Medical College

Zhigang Ren ( $\nabla$ fccrenzg@zzu.edu.cn )

Zhengzhou University First Affiliated Hospital

\section{Research}

Keywords: Paraquat poisoning, Risk model, Machine learning, In-hospital death, Prognosis

Posted Date: February 25th, 2020

DOI: https://doi.org/10.21203/rs.2.24492/v1 
License: (c) (i) This work is licensed under a Creative Commons Attribution 4.0 International License. Read Full License

Version of Record: A version of this preprint was published at Scientific Reports on January 8th, 2021. See the published version at https://doi.org/10.1038/s41598-020-80371-5. 


\section{Abstract}

Background: Acute paraquat (PQ) poisoning is characterized by multi-organ failure and lacking effective therapies. Therefore, identifying risk factors and developing model that could predict early prognosis for patients with PQ poisoning is of great importance.

Methods: This was a retrospective cohort study employed with patients suffered from acute PQ poisoning ( $n=1199)$. Patients ( $\mathrm{n}=913$ ) with PQ poisoning from 2011 to 2018 were randomly divided into 2 mutually exclusive groups: training (609 patients) and test (304 patients). Another 2 external cohorts containing 207 cases from Zhengzhou 2019 were used as validation from different time and 79 from Shenyang as validation from different site. Risk factors were identified by a logistic model with Markov Chain Monte Carlo (MCMC) simulation and further evaluated by a latent class analysis. The prediction score of this model was developed based on the training sample and was further evaluated using the testing and validation samples.

Results: Eight risk factors including age, ingestion volume, CK-MB, platelet (PLT), white blood cell (WBC), neutrophil counts (N), gamma-glutamyl transferase (GGT) and serum creatinine (Cr) were identified as in dependent risk indicators of in-hospital death events. The risk model had a C statistic of 0.895 (95\% Cl 0.855-0.928), 0.891 (95\% Cl 0.848-0.932) and 0.829 (95\% Cl 0.4551.000 ) and a predictive range of $4.6 \%-98.2 \%, 2.3 \%-94.9 \%$ and $0 \%-12.5 \%$ for the test, validation_time and validation_site group, respectively. In the training group, the risk model classified $18.4 \%, 59.9 \%$ and $21.7 \%$ of patients into the high, average and lowrisk groups, with corresponding probabilities of $0.985,0.365$, and 0.03 for in-hospital death events.

Conclusion: Eight risk factors were identified in this study. And we developed and evaluated a simple risk model to predict the prognosis of patients with acute $P Q$ poisoning. This simple and reliable risk score system could be helpful in recognizing highrisk patients and reducing in-hospital death rate due to $P Q$ poisoning.

\section{Introduction}

As a non-selective contact herbicide, paraquat $(\mathrm{PQ})$ is environmental harmless and predominantly used in developing agricultural countries [1,2]. However, it is lethal to human and animals when ingested orally and there are still no effective and specific antidotes for PQ. Patients with acute $P Q$ poisoning usually died within several days to weeks after confirmed exposure due to hypoxemia or multiple organs failure, which causes a considerable economic burden and increased medical resource use for families and countries [3-6]. Therefore, for patients, especially in critically ill patients, clinical outcome evaluation and risk assessment in a timely manner is crucial and essential for medical resources using wisely, which has become a public health problem concerned by doctors and patients, as well as social security agency.

To our knowledge, several prognostic score systems have been reported to predict the clinical outcomes of patients with PQ poisoning, the major ones being the Acute Physiology and Chronic Health Evaluation II (APACHE II) score [7], Sequential Organ Failure Assessment (SOFA) score [8], the severity index of PQ poisoning (SIPP) [9], Poisoning Severity Score (PSS) [10] and some equations and nomograms based on large cohort study [11-13]. Most of them are more suitable for critically ill patients instead of minimally poisoned or early-stage patients who showed mild symptoms. Moreover, it is highly possible for these score systems failed to predict mortality and conduct risk assessment for PQ poisoned patients instantly because of their difficult calculation or unavailable laboratory tests, which cannot meet emergency work demand. Thus, the establishment of an effective, simple and universal predictive model based on common laboratory items would be of great help in risk stratification and therapeutic regimen adjustment for patients with acute PQ poisoning in all stages.

Accordingly, on the basis of 1199 patients with PQ-poisoning from two large academic hospitals in china with sufficient patients' sources, we established and evaluated a simple risk model by identifying significant clinical risk indicators to predict in-hospital death. Data from our study were collected from medical records of acute PQ-poisoned patients in different time and different region. The end point of this research was patients' in-hospital death before discharge. This study is well designed and easily conducted to generate tools to predict in-hospital death by a combination of simple and clinically relevant variables.

\section{Methods}




\section{Study samples and data source}

The PQ poisoning study enrolled 932 patients from January 1, 2011 to December 31,2018. Patient information was collected during the first two months in 2019. Extra 207 samples from Zhengzhou 2019 and 79 from Shenyang Shengjing hospital were collected as external validations. We excluded patients with missing values of $\operatorname{sex}(n=11)$ and in-hospital death $(n=22)$. The final sample included 913 unique patients who were randomly divided into 2 mutually exclusive samples for training (66.7\% [609 patients]) and test (33.3\% [304 patients]). The training sample was used to select risk factors and the test sample was used for evaluation. External 207 samples from Zhengzhou 2019 were used as validation from different time and 79 from Shenyang as validation from different hospital (Fig. 1). The Institutional Review Board of the First Affiliated Hospital of Zhengzhou University (2017-XY-002) approved this study and exemption of the patient's consent.

\section{Potential Risk Factors And Outcome}

We selected candidate risk factors that were clinically meaningful, were reliable and easily collected, occurred at a frequency more than $1 \%$. Initial factors include patient demographic characteristics (age, sex), ingestion hour, ingestion volume and laboratory tests (BUN, CK, CK-MB, GGT, MPV, PCT, PLT, WBC, N, AST, ALT, and Cr). An additional file showed the detailed distribution information of variables in the 1199 samples (Table S). To facilitate the calculation of risk score, we examined the nonlinear relationship of each continuous factor with the outcome and categorized it by a cut-off point taking into account both in-hospital death rates and sample sizes (Additional file: Figure S). Factors with missing values were imputed using multiple imputations with 10 imputations. The final imputed value was an average of the 10 imputations. Rates of missing ranged from $1.1 \%$ (age) to $13.3 \%$ (CK-MB). The outcome is in-hospital death.

\section{Statistical Analyses}

\section{Risk factor selection and evaluation}

We fit a logistic model with Markov Chain Monte Carlo (MCMC) simulation using the training cohort that containing all candidate risk factors. Subsequently, a posterior probability for each factor was further calculated to assess the association strength between risk factor and the clinical outcome [14, 15]. Generally, the factor with a posterior probability $>0.95$ (or $<0.05$ for factors with estimates $<0.0$ ) was thought to be significant for predicting the prognosis and finally included in the risk factor list [16]. Next, based on the selected risk factors by the MCMC method, we established the final risk model to predict the outcome by fitting a logistic model to the training group.

The following indicators were calculated to assess the risk model performance: the Harrell C statistic to evaluate the overall predictive accuracy $[17,18]$, the McFadden R square to evaluate explained variation [19], and the Hosmer-Lemeshow goodnessof-fit test to evaluate calibration [20]. The discrimination was then assessed among the observed outcomes in strata defined by deciles of the predictive probabilities. As described in previous publications [15], according to the deciles, patients in the training group were divided into 5 mutually exclusive risk classes, ranking the classes from lowest risk (class 1 ) to highest risk (class 5 ) for evaluation.

Further, data from training group were used to evaluate the selected risk factors. Latent class analysis was conducted by an unsupervised machine learning algorithm that does not require an outcome [14]. Generally, if the selected risk factors are significantly associated with the clinical outcome, they will have the ability to assign patients to risk class with unsupervised learning algorithms. Therefore, we performed latent class analysis to classify patients into 5 mutually exclusive classes and ranked them from lowest risk (class 1) to highest risk (class 5) based on the observed outcome. Then 5 classes were selected to align with the decile-specific classes based on the risk model described previously. We calculated a Spearman correlation coefficient between the risk classes based on the risk model and the risk classed based on latent class analysis. A high coefficient indicates good agreement between the 2 classified results, which provides information on the robustness of the selected risk factors. 
Furthermore, we revalidated this risk model by comparing the performance in the training group with the test and another 2 independent validation groups.

\section{Risk Score}

A simple risk score was constructed to facilitate the use of the selected risk factors and the risk model for each patient based on the regression coefficients estimated from the training group. The coefficient of risk factor was divided by the sum of all coefficients in the model to calculate the points of each risk factor, multiplying by 100 , and rounding to the nearest integer. Patients were stratified into 3 risk groups according to the distribution of the risk score: low (<25\%), average $(25 \%-75 \%)$, and high (> 75\%).

Statistical analyses were conducted using SAS statistical software version 9.4 (SAS institute Inc.). The latent class analysis was performed using the PROC LCA procedure, (version 1.3.2 beta). The nonlinear relationship was assessed using the PROC GAM procedure. The study followed the Transparent Reporting of a Multivariable Prediction Model for Individual Prognosis or Diagnosis (TRIPOD) reporting guideline. Each of the 22 items of the TRIPOD statement was addressed.

\section{Results}

\section{Study sample}

A total of 1199 (609 training, 304 test, 207 validation by time, and 79 validation by site) participants were enrolled. The mean (SD) age was 35.1 (16.4) years old and 599 patients (50.0\%) were female. There were some differences in basic clinical characteristics of patients across training, test and 2 validation samples. And the difference between the training group and the test and validation groups demonstrates the predictability and extrapolation of the model (Table 1). 
Table 1

Patient characteristics by sample groups.

\begin{tabular}{|c|c|c|c|c|c|c|}
\hline Characteristics & Aggregate & Training & Test & Validation_time & Validation_site & P_VALUE \\
\hline Total & 1199 & 609 & 304 & 207 & 79 & \\
\hline inhos_death n(\%) & $454(37.9)$ & $247(40.6)$ & $117(38.5)$ & $87(42.0)$ & $3(3.8)$ & $<0.0001$ \\
\hline female $n(\%)$ & $599(50.0)$ & $297(48.8)$ & $172(56.6)$ & $93(44.9)$ & $37(46.8)$ & 0.0444 \\
\hline $\begin{array}{l}\text { age } \\
\text { mean(std) median(q1,q3) }\end{array}$ & $\begin{array}{l}35.1(16.4) \\
34(23,47)\end{array}$ & $\begin{array}{l}34.7(16.5) \\
34(23,46)\end{array}$ & $\begin{array}{l}34(16.2) \\
30.5(23,46)\end{array}$ & $\begin{array}{l}36.1(17.2) \\
34(25,48)\end{array}$ & $\begin{array}{l}40.1(12.8) \\
40(29,48)\end{array}$ & 0.0104 \\
\hline age_It_50 n(\%) & $963(80.3)$ & 495 (81.3) & $246(80.9)$ & $159(76.8)$ & $63(79.7)$ & 0.5614 \\
\hline age_ge_50 n(\%) & $236(19.7)$ & $114(18.7)$ & $58(19.1)$ & $48(23.2)$ & $16(20.3)$ & 0.5614 \\
\hline $\begin{array}{l}\text { ALT1 } \\
\text { mean(std) median(q1,q3) }\end{array}$ & $\begin{array}{l}46.8(114.6) \\
18(12,33)\end{array}$ & $\begin{array}{l}46.8(102.2) \\
18(12,34)\end{array}$ & $\begin{array}{l}40.2(81.1) \\
16(12,30.5)\end{array}$ & $\begin{array}{l}62.1(187.4) \\
19(12,41)\end{array}$ & $\begin{array}{l}32(32.5) \\
20(13,32)\end{array}$ & 0.4286 \\
\hline ALT1_It_100 & $1073(89.5)$ & $546(89.7)$ & $274(90.1)$ & $179(86.5)$ & $74(93.7)$ & 0.3053 \\
\hline ALT1_ge_100 & $126(10.5)$ & $63(10.3)$ & $30(9.9)$ & 28 (13.5) & $5(6.3)$ & 0.3053 \\
\hline AST1 & $\begin{array}{l}57.1(139) \\
23(17,39)\end{array}$ & $\begin{array}{l}58.9(146) \\
23(18,41)\end{array}$ & $\begin{array}{l}53.3(117.6) \\
23(17,37.5)\end{array}$ & $\begin{array}{l}64.7(168.3) \\
23.2(18,41)\end{array}$ & $\begin{array}{l}38.3(39.3) \\
22(17,32)\end{array}$ & 0.3409 \\
\hline AST1_It_60 & $982(81.9)$ & $498(81.8)$ & 249 (81.9) & $170(82.1)$ & $65(82.3)$ & 0.9992 \\
\hline AST1_ge_60 & $217(18.1)$ & $111(18.2)$ & $55(18.1)$ & $37(17.9)$ & $14(17.7)$ & 0.9992 \\
\hline BUN1 & $\begin{array}{l}6.8(5.8) \\
5.2(4,7.4)\end{array}$ & $\begin{array}{l}6.8(5.5) \\
5.4(4,7.7)\end{array}$ & $\begin{array}{l}6.7(5.5) \\
5(3.9,7.3)\end{array}$ & $\begin{array}{l}7.1(7.2) 5.2(4, \\
7.3)\end{array}$ & $\begin{array}{l}6.1(5.4) \\
4.9(4.1,6.5)\end{array}$ & 0.3161 \\
\hline BUN1_It_10 & 1045 (87.2) & $530(87.0)$ & 259 (85.2) & $180(87.0)$ & $76(96.2)$ & 0.0774 \\
\hline BUN1_ge_10 & $154(12.8)$ & $79(13.0)$ & 45 (14.8) & $27(13.0)$ & $3(3.8)$ & 0.0774 \\
\hline CK_MB1 & $\begin{array}{l}29.4(38.6) \\
20.2(12.9, \\
33.6)\end{array}$ & $\begin{array}{l}31(39.6) \\
21(13.6,36)\end{array}$ & $\begin{array}{l}31.3(40.3) \\
20.9(13.8, \\
32.7)\end{array}$ & $\begin{array}{l}28.6(37.7) \\
19.1(12.3 \\
33.3)\end{array}$ & $\begin{array}{l}12.1(14) 3.8(2 \\
25.5)\end{array}$ & $<0.0001$ \\
\hline CK_MB1_It_50 & $1054(87.9)$ & $518(85.1)$ & $269(88.5)$ & 189 (91.3) & $78(98.7)$ & 0.0013 \\
\hline CK_MB1_ge_50 & $145(12.1)$ & $91(14.9)$ & 35 (11.5) & $18(8.7)$ & $1(1.3)$ & 0.0013 \\
\hline CR_1 & $\begin{array}{l}108.4(124.7) \\
66(51,117)\end{array}$ & $\begin{array}{l}108(108.2) \\
67(51,123)\end{array}$ & $\begin{array}{l}109(123.1) \\
66(49.5 \\
117)\end{array}$ & $\begin{array}{l}114.4(152.9) \\
66(52,105.5)\end{array}$ & $\begin{array}{l}92.6(163.1) \\
60.7(51.4 \\
81.6)\end{array}$ & 0.4877 \\
\hline CR_1_It_150 & 985 (82.2) & $492(80.8)$ & 251 (82.6) & 168 (81.2) & 74 (93.7) & 0.0441 \\
\hline CR_1_ge_150 & $214(17.8)$ & $117(19.2)$ & $53(17.4)$ & 39 (18.8) & $5(6.3)$ & 0.0441 \\
\hline DBiL1 & $\begin{array}{l}10.2(22.3) \\
5.1(3.4,7.7)\end{array}$ & $\begin{array}{l}11(23.5) \\
5.1(3.6,7.8)\end{array}$ & $\begin{array}{l}9.1(14.1) \\
5.4(3.5,8.1)\end{array}$ & $\begin{array}{l}11.2(30.7) \\
4.8(3.3,6.8)\end{array}$ & $\begin{array}{l}6.4(6.3) 4(2.9 \\
6.1)\end{array}$ & 0.0033 \\
\hline DBiL1_It_20 & $1080(90.1)$ & $541(88.8)$ & 277 (91.1) & $188(90.8)$ & 74 (93.7) & 0.4418 \\
\hline DBiL1_ge_20 & 119 (9.9) & $68(11.2)$ & 27 (8.9) & $19(9.2)$ & $5(6.3)$ & 0.4418 \\
\hline GGT1 & $\begin{array}{l}50.7(95.8) \\
21(14,47.8)\end{array}$ & $\begin{array}{l}50.6(101.3) \\
19(13.4 \\
37.3)\end{array}$ & $\begin{array}{l}43.4(74.1) \\
18.5(13.4 \\
40)\end{array}$ & $\begin{array}{l}56(119.1) \\
23.9(14,50)\end{array}$ & $\begin{array}{l}65.6(42.1) \\
61.4(47,79.2)\end{array}$ & $<0.0001$ \\
\hline GGT1_It_150 & 1134 (94.6) & $569(93.4)$ & 291 (95.7) & $196(94.7)$ & 78 (98.7) & 0.1714 \\
\hline GGT1_ge_150 & $65(5.4)$ & $40(6.6)$ & $13(4.3)$ & $11(5.3)$ & $1(1.3)$ & 0.1714 \\
\hline
\end{tabular}




\begin{tabular}{|c|c|c|c|c|c|c|}
\hline Characteristics & Aggregate & Training & Test & Validation_time & Validation_site & P_VALUE \\
\hline MPV & $\begin{array}{l}9.2(3.6) \\
8.9(8,9.9)\end{array}$ & $\begin{array}{l}9.1(1.5) \\
9(8.1,9.8)\end{array}$ & $\begin{array}{l}9.2(1.6) \\
8.9(8,10.2)\end{array}$ & $\begin{array}{l}9.6(8.1) \\
8.6(7.9,9.7)\end{array}$ & $\begin{array}{l}9.2(1.1) \\
9.2(8.3,9.9)\end{array}$ & 0.0487 \\
\hline MPV_It_10d5 & 1004 (83.7) & $518(85.1)$ & $238(78.3)$ & 179 (86.5) & 69 (87.3) & 0.0256 \\
\hline MPV_ge_10d5 & 195 (16.3) & 91 (14.9) & $66(21.7)$ & $28(13.5)$ & $10(12.7)$ & 0.0256 \\
\hline N1 & $\begin{array}{l}83.8(13.4) \\
88.9(79.3 \\
92.5)\end{array}$ & $\begin{array}{l}84.3(13.2) \\
89.2(80.2, \\
92.6)\end{array}$ & $\begin{array}{l}84.3(13.8) \\
89.5(81.1 \\
92.6)\end{array}$ & $\begin{array}{l}82.7(13.7) \\
87.9(76.2, \\
92.7)\end{array}$ & $\begin{array}{l}81(11.1) \\
84.1(74.2,89)\end{array}$ & 0.0007 \\
\hline N1_It_80 & $310(25.9)$ & $149(24.5)$ & $68(22.4)$ & $60(29.0)$ & $33(41.8)$ & 0.0029 \\
\hline N1_ge_80 & $889(74.1)$ & $460(75.5)$ & $236(77.6)$ & 147 (71.0) & $46(58.2)$ & 0.0029 \\
\hline РCT & $\begin{array}{l}0.2(0.1) \\
0.2(0.1,0.2)\end{array}$ & $\begin{array}{l}0.1(0.1) \\
0.1(0.1,0.2)\end{array}$ & $\begin{array}{l}0.1(0.1) \\
0.1(0.1,0.2)\end{array}$ & $\begin{array}{l}0.2(0.1) \\
0.2(0.1,0.2)\end{array}$ & $\begin{array}{l}0.1(0.1) \\
0.1(0.1,0.2)\end{array}$ & $<0.0001$ \\
\hline PCT_It_0d2 & 903 (75.3) & 483 (79.3) & $240(78.9)$ & 119 (57.5) & $61(77.2)$ & $<0.0001$ \\
\hline PCT_ge_0d2 & $296(24.7)$ & $126(20.7)$ & 64 (21.1) & $88(42.5)$ & $18(22.8)$ & $<0.0001$ \\
\hline PLT & $\begin{array}{l}179.4(90) \\
172(113 \\
236)\end{array}$ & $\begin{array}{l}\text { 173.6(87.7) } \\
\text { 165(109, } \\
226)\end{array}$ & $\begin{array}{l}\text { 170.2(90.5) } \\
157(107, \\
218)\end{array}$ & $\begin{array}{l}218.4(88) \\
211(167,271)\end{array}$ & $\begin{array}{l}158.1(84) \\
161(80,220)\end{array}$ & $<0.0001$ \\
\hline PLT_It_80 & 155 (12.9) & $79(13.0)$ & 49 (16.1) & $11(5.3)$ & $16(20.3)$ & 0.0006 \\
\hline PLT_ge_80 & 1044 (87.1) & $530(87.0)$ & 255 (83.9) & 196 (94.7) & $63(79.7)$ & 0.0006 \\
\hline WBC1 & $\begin{array}{l}14.1(8) \\
11.8(8.6 \\
17.3)\end{array}$ & $\begin{array}{l}\text { 14.4(8.4) } \\
11.9(8.6, \\
18.5)\end{array}$ & $\begin{array}{l}14.4(7.9) \\
12.4(9.2 \\
17.7)\end{array}$ & $\begin{array}{l}13.2(7.1) \\
11.1(8.3,16.5)\end{array}$ & $\begin{array}{l}12.3(6.5) \\
10.6(8.3,14.4)\end{array}$ & 0.0373 \\
\hline WBC1_It_20 & 979 (81.7) & $484(79.5)$ & $250(82.2)$ & 173 (83.6) & 72 (91.1) & 0.0642 \\
\hline WBC1_ge_20 & $220(18.3)$ & 125 (20.5) & $54(17.8)$ & $34(16.4)$ & $7(8.9)$ & 0.0642 \\
\hline LDH1 & $\begin{array}{l}279(151) \\
236.1(205 \\
299.3)\end{array}$ & $\begin{array}{l}289.4(102.8) \\
273.3(218.4 \\
333)\end{array}$ & $\begin{array}{l}310.8 \\
(165.4) \\
259.7(235.5, \\
320.5)\end{array}$ & $\begin{array}{l}356.2(253.3) \\
265.1(221.7 \\
363.3)\end{array}$ & $\begin{array}{l}219.3(58.1) \\
209.6(182.8 \\
234.8)\end{array}$ & $<0.0001$ \\
\hline LDH1_It_240 & 1101 (91.8) & 579 (95.1) & 277 (91.1) & $184(88.9)$ & $61(77.2)$ & $<0.0001$ \\
\hline LDH1_ge_240 & $98(8.2)$ & $30(4.9)$ & $27(8.9)$ & $23(11.1)$ & $18(22.8)$ & $<0.0001$ \\
\hline ingestion_volume & $\begin{array}{l}61.1(67.5) \\
40(20,80)\end{array}$ & $\begin{array}{l}65.1(69.9) \\
40(20,100)\end{array}$ & $\begin{array}{l}55.8(63.4) \\
30(15,80)\end{array}$ & $\begin{array}{l}61.5(72) 30(15 \\
80)\end{array}$ & $\begin{array}{l}49.8(47.3) \\
40(20,60)\end{array}$ & 0.2922 \\
\hline Ingestion_volume_It_100 & $927(77.3)$ & $452(74.2)$ & $244(80.3)$ & $161(77.8)$ & 70 (88.6) & 0.0141 \\
\hline Ingestion_volume_ge_100 & $272(22.7)$ & $157(25.8)$ & $60(19.7)$ & $46(22.2)$ & $9(11.4)$ & 0.0141 \\
\hline LOS & $\begin{array}{l}7.3(6.8) 5(2, \\
11)\end{array}$ & $\begin{array}{l}7.4(7) 5(2, \\
10)\end{array}$ & $\begin{array}{l}7.9(6.9) 6(2, \\
11.5)\end{array}$ & $\begin{array}{l}6.9(6.1) 5.1(2 \\
10.7)\end{array}$ & $5.5(5.8) 3(1,8)$ & 0.0045 \\
\hline
\end{tabular}

\section{In-hospital Death Events}

The rates of in-hospital death were $40.6 \%(95 \% \mathrm{Cl}, 36.6 \%-44.6 \%)$ and $38.5 \%(95 \% \mathrm{Cl}, 33.0 \%-44.2 \%)$ respectively for the training and test samples. The average in-hospital death was $37.9 \%$. The median (interquartile range [IQR]) length of hospital stay was 5 (2-11) days (Table 1). 


\section{Risk Factor Selection And Test}

The MCMC simulation selected 8 candidate factors with a posterior probability of at least 0.95 (Table 2), including age, ingestion volume, CK-MB, PLT, WBC, N, GGT and $\mathrm{Cr}$ (Fig. 2). The risk model based on the 8 risk factors and the training group demonstrated good discrimination, calibration and fit. The overall C statistic was $0.926(95 \% \mathrm{Cl}, 0.891-0.924)$ for the risk model (Fig. 3). The mean observed in-hospital death ranged from 3.3\% in the lowest predicted quintile to $99.2 \%$ in the highest predicted quintile, a range of $95.9 \%$ and the explained variation was 0.4999 (Fig. 4). Moreover, the $P$ value of the Hosmer-Lemeshow goodness-of-fit test was 0.4262 in the training group and 0.9078 in the test group, indicating that the model fitted well with the observed cohort (Fig. 5).

Table 2

Final risk prediction model for in-hospital death event based on training group

\begin{tabular}{|llll|}
\hline Variable & Estimate & StdErr & Score_R* \\
\hline WBC1_ge_20 & 2.743299958 & 0.42703288 & 19 \\
\hline CK_MB1_ge_50 & 2.35461446 & 0.6455247 & 16 \\
\hline N1_ge_80 & 2.162847223 & 0.438185092 & 15 \\
\hline Ingestion_volume_ge_100 & 2.018090939 & 0.3082356 & 14 \\
\hline CR_1_ge_150 & 1.638231401 & 0.356155153 & 11 \\
\hline age_ge_50 & 1.427619459 & 0.329849932 & 9 \\
\hline GGT1_ge_150 & 1.387451403 & 0.566479088 & 6 \\
\hline PLT_It_80 & 0.921076865 & 0.331850564 & 100 \\
\hline total & & & 10 \\
\hline $\begin{array}{l}\text { *Risk scores were calculated by dividing a risk factor's coefficient by the sum of all coefficients, multiplying by } 100, \text { and } \\
\text { rounding to the nearest integer. }\end{array}$
\end{tabular}

Additionally, model performance in the test sample was comparable to that in the training sample. The overall C Statistic was 0.895 (95\% Cl $0.855-0.928$ ) in the test sample (Fig. 3). The rate of observed in-hospital death ranged from $4.6 \%$ in the lowest predicted quintile to $98.2 \%$ in the highest predictive quintile, the explained variation was 0.4182 for test group (Fig. 4).

Furthermore, in the latent class analysis, 609 patients in the training group were assigned into 5 classes based on the combination of the 8 risk factors (Fig. 6). For this analysis the area under the ROC curve was $0.877(95 \% \mathrm{Cl}, 0.832-0.906)$, and the mean observed outcome rate ranged from $0.0 \%$ in the lowest rating group to $99.2 \%$ in the highest rating group. The spearman correlation coefficient between the predicted quintile based on the Logistic model and the latent class analysis was $0.754(95 \% \mathrm{Cl}, 0.715-0.874)$.

\section{Risk Score System}

We observed that the risk factor-specific points ranged from $19(\mathrm{WBC}>=20)$ to 6 (PLT < 80) (Table 2). WBC > = 20, CK-MB >=50, $\mathrm{N}>=80$, Ingestion volume $>=100$, and $\mathrm{Cr}>=150$ were the top 5 factors with an odds ratio more than 5.0 (Fig. 2). The training group has mean (SD) risk score of 26.6 (21.6). For the test group, the mean (SD) score was 24.8 (21.1). In addition, in the training sample, $18.4 \%, 59.9 \%$ and $21.7 \%$ of patients were stratified into the high, average and low-risk groups, with a corresponding probability of $0.985,0.365$, and 0.03 for in-hospital death respectively (Fig. 7). And the stratification for the test sample was not markedly different from that of the training group (Fig. 7, Table 3). 
Table 3

Patient risk stratification based on risk score

\begin{tabular}{|c|c|c|c|c|c|c|c|c|}
\hline \multirow[t]{2}{*}{$\begin{array}{l}\text { Risk } \\
\text { group }\end{array}$} & \multicolumn{2}{|c|}{ Training $(n=609)$} & \multicolumn{2}{|c|}{ Test $(n=304)$} & \multicolumn{2}{|c|}{ Validation_time $(n=207)$} & \multicolumn{2}{|c|}{$\begin{array}{l}\text { Validation_site }(n= \\
79)\end{array}$} \\
\hline & $\begin{array}{l}\text { Patients } \\
\#(\%)\end{array}$ & $\begin{array}{l}\text { Probability of } \\
\text { in-hospital } \\
\text { death, } \\
\text { mean (std) }\end{array}$ & $\begin{array}{l}\text { Patients } \\
\#(\%)\end{array}$ & $\begin{array}{l}\text { Probability of } \\
\text { in-hospital } \\
\text { death, } \\
\text { mean(std) }\end{array}$ & $\begin{array}{l}\text { Patients } \\
\#(\%)\end{array}$ & $\begin{array}{l}\text { Probability } \\
\text { of in- } \\
\text { hospital } \\
\text { death, } \\
\text { mean(std) }\end{array}$ & $\begin{array}{l}\text { Patients } \\
\#(\%)\end{array}$ & $\begin{array}{l}\text { Probability } \\
\text { of in- } \\
\text { hospital } \\
\text { death, } \\
\text { mean(std) }\end{array}$ \\
\hline $\begin{array}{l}\text { High } \\
(46+)\end{array}$ & $\begin{array}{l}112 \\
(18.4)\end{array}$ & $0.98(0.02)$ & $\begin{array}{l}47 \\
(15.5)\end{array}$ & $0.99(0.02)$ & $28(13.5)$ & $0.98(0.02)$ & $2(2.5)$ & $\begin{array}{l}0.97 \\
(0.04)\end{array}$ \\
\hline $\begin{array}{l}\text { Average } \\
(15-45)\end{array}$ & $\begin{array}{l}365 \\
(59.9)\end{array}$ & $0.36(0.28)$ & $\begin{array}{l}189 \\
(62.2)\end{array}$ & $0.34(0.25)$ & $123(59.4)$ & $0.38(0.27)$ & $\begin{array}{l}48 \\
(60.8)\end{array}$ & $\begin{array}{l}0.33 \\
(0.24)\end{array}$ \\
\hline $\begin{array}{l}\text { Low }(0- \\
14)\end{array}$ & $\begin{array}{l}132 \\
(21.7)\end{array}$ & $0.03(0.02)$ & $\begin{array}{l}68 \\
(22.4)\end{array}$ & $0.02(0.02)$ & $56(27.1)$ & $0.03(0.03)$ & $\begin{array}{l}29 \\
(36.7)\end{array}$ & $\begin{array}{l}0.03 \\
(0.02)\end{array}$ \\
\hline
\end{tabular}

\section{Risk Model Validation}

For the validation groups, the in-hospital death events rates were $42.0 \%(95 \% \mathrm{Cl}, 35.2 \%-49.1 \%)$ and $3.8 \%(95 \% \mathrm{Cl}, 0.8 \%-10.7 \%)$ for validation_time and validation_site respectively (Table 1). The observed in-hospital death rates ranged from $2.3 \%, 0.0 \%$ in the lowest predicted quintile to $94.9 \%, 12.5 \%$ in the highest predictive quintile, the explained variation was 0.3798 , and 0.2421 for validation by time and validation by site respectively. Meanwhile, the overall C Statistic was $0.891(95 \% \mathrm{Cl} 0.848-0.932)$ and 0.827 (95\% Cl 0.455-1.000) (Fig. 3) and the p value of Hosmer and Lemeshow's Goodness of Fit Test was 0.9671 and 0.9999 for the two independent groups (Fig. 5).

For the validation groups, the mean (SD) of risk scores were 23.5 (19.7), 16.3 (14.1), validation by time and validation by site group, respectively. In the validation_time group, $27.1 \%, 59.4 \%$ and $13.5 \%$ were classified into the low-, average- and high-risk groups, respectively, with corresponding probabilities of $0.98,0.38$ and 0.03 for in-hospital death events (Fig. 7 and Table 3 ). In the validation_site group, $36.7 \%, 60.8 \%$ and $2.5 \%$ were classified into low-, average- and high-risk groups, with a correspondence probability of $0.03,0.33$ and 0.97 respectively. The probabilities for in-hospital death events were identical to the training group (Fig. 7 and Table 3).

\section{Discussion}

In this large study, we first evaluated 609 patients with acute $P Q$ poisoning and revealed eight prognostic variables for survival after PQ poisoning, including WBC, CK-MB, N, Ingestion Volume, Cr, Age, GGT and PLT. Next, a random forest risk model and score system based on the above risk factors was established to predict the in-hospital death for patients diagnosed with $P Q$ poisoning. Furthermore, our model was further verified both in internal and external validation cohorts. The risk factors identified in this model were based on data from medical records and easily collection and ready availability when the patients discharged. Meanwhile, the statistical algorithms are robust in this study. Importantly, the risk predictive model and its corresponding risk scores system may help clinicians distinguish patients with higher risk of in-hospital death after PQ poisoning.

Our research, on the basis of information selected from medical records of PQ poisoned patients, presents a large study that predict the in-hospital death. $\mathrm{PQ}$ poisoning is a major cause of fatal poisoning in most regions of Asia nations. $\mathrm{PQ}$ could cause multiple organ failure including severe pulmonary fibrosis, which is the main cause of death in paraquat poisoning [21, 22]. Although efficacious therapeutic strategies for acute $P Q$ poisoning management have been extensively investigated, early prognosis of $P Q$ poisoning remains unpleasant $[23,24]$. Generally, the clinical outcomes of acute $P Q$ poisoning are associated with the ingested dose. Previous studies have reported that plasma PQ concentrations are powerful tool for patients with $P Q$ 
poisoning to predict clinical prognosis [25, 26]. However, it is not available in most local hospitals to monitor serum PQ concentration. Moreover, the poison intaking amount is difficult to accurately assess, particularly in patients presenting with confusion. Identification the risk factors that could influence the clinical outcome in $P Q$ poisoning in early stage may improve the early diagnosis and rapid medical intervention for patients who are at the highest risk of death. Thus, establishing a reliable, simple and universal risk predictive model based on available laboratory parameters is urgently need to judge the early prognosis of PQ-poisoned patients. Comparing with prior studies that only focused on independent clinical indicator, in the present work, clinical indicators included in this model was routine and inexpensive, so almost every hospital can easily acquire the data. Meanwhile, validating and classifying the risk of in-hospital death based on multi-clinical index model is the most important innovation, which would be helpful in risk stratification and therapeutic regimen adjustment for acute PQ-poisoned patients in the field of emergency medical research.

The strength of the association between the risk factors and the clinical outcome was evaluated using the MCMC algorithm. Clinical data from 2011 to 2018 were used to construct and evaluate this novel noninvasive predictive risk model. Further, based on another two external independent cohorts from different time and different site for scoring system revalidation, our research had better predictive accuracy compared with other studies. Previous studies have recognized the SIPP as the most potentially valuable prognostic indicator for PQ-poisoned patients [9, 27]. The lack of availability of these indicators in many hospitals makes it hard to apply the proposed scoring system on a daily basis, which would also limit the accurate evaluation of poisoning severity. The APACHE II scoring system is also applied in clinical to evaluate the prognosis of PQ-poisoned patients $[28,29]$. However, this scoring system might underestimate mortality in critically ill patients. Interestingly, our predictive risk model was established on the clinical indicators at baseline of PQ-poisoned patients. It is convenient to use and exclude the hopeless or minimally poisoned patients from needless aggressive therapy.

The risk factors in our study were identified using a large cohort and further tested and validated consistent with the training results. Hidden factors applied for constructing a risk model may affect the efficiency of the ultimate model in many aspects. Data for an effective risk factor ought to be conveniently collected, stabilized by clinical illustration, and widely received during hospitalization as well as at discharge. Notably, the 8 risk factors selected in our study met the whole criteria. Our risk model includes age, ingestion volume, CK-MB, PLT, WBC, N, GGT and Cr. Previous publications demonstrated that WBC and neutrophil counts were essential indexes for the prognosis of $P Q$ poisoning $[30,31]$. $\mathrm{Cr}$, one of the factors associated with poor prognosis from the risk model, could be induced by direct oxidative injury in renal tubules [32]. Elevated serum creatinine level was closely associated with acute kidney injury (AKI). And PQ-patients with AKI had higher mortality risk than those normal [33]. Through risk stratification, we found that in the training group, the proportion of patients at high risk or general risk of experiencing inhospital death after PQ poisoning diagnosis was $98 \%$ and $36 \%$, respectively. The 8 risk factors could aid clinicians making a reasonable medical intervention that may improve prognosis and reduce unnecessary treatment. To some extent, the economic burden on healthcare might be greatly relieved with the improvement of patients' clinical prognosis and the reduction of inhospital death.

In conclusion, the simple 8-factor risk model showed good reliability and validity and provided a basis for clinicians to identify high risk patients after $P Q$ poisoning. The severity and early prognosis of $P Q$ poisoned patients can be precisely recognized by this model. It assists clinicians make better-targeted, evidence-based decisions for in-hospital PQ poisoning management.

\section{Conclusion}

The early clinical outcome for PQ-poisoned patients has thus far been difficult to evaluate. The novel risk model we established is more convenient to use and exclude the hopeless or minimally poisoned patients from needless aggressive therapy than conventional scoring systems such as APACHE II, SOFA and SIPP. This risk score system is suitable for clinical use in recognizing high-risk $\mathrm{PQ}$-poisoned patients and predicting early in-hospital death.

\section{Abbreviations}


PQ: paraquat; MCMC: Markov Chain Monte Carlo; APACHE II: Acute Physiology and Chronic Health Evaluation II; SOFA: Sequential Organ Failure Assessment; SIPP: the severity index of PQ poisoning; PSS: Poisoning Severity Score; PLT: platelet; WBC: white blood cell; N: neutrophil counts; GGT: gamma-glutamyl transferase; Cr: serum creatinine.

\section{Declarations}

\section{Acknowledgements}

We thank the clinical doctors from the Precision Medicine Center and Emergency Department of the First Affiliated Hospital of Zhengzhou University, who have participated in this study.

\section{Authors' Contributions}

ZR, YG and YL designed the study. YG, TL, DY, YW, ZX, LH, YZ, GD, CS, LC and SL collected clinical data; ZR, YG and GC analyzed the data and established the model; ZR, LL, YG and $\mathrm{HZ}$ wrote the manuscript. All authors reviewed and approved the manuscript.

\section{Funding}

This work was supported by the National Natural Science Foundation of China (81701893), National S\&T Major Project of China (2018ZX10301201-008), Key Scientific Research Projects of Higher Education Institutions in Henan Province (20A320046 and 20A320056), Joint Construction Project of Henan Province Medical S\&T Research (SB201901006).

\section{Availability of data and materials}

The datasets used and/or analyzed during the current study are available from the corresponding author on reasonable request.

\section{Ethics approval and consent to participate}

The study protocol complies with the Declaration of Helsinki. The Institutional Review Board of the First Affiliated Hospital of Zhengzhou University (2017-XY-002) approved this study and exemption of the patient's consent.

\section{Consent for publication}

Not applicable

\section{Competing interests}

The authors declare that they have no competing interests.

\section{Author details}

${ }^{1}$ Emergency Department, the First Affiliated Hospital of Zhengzhou University, Zhengzhou 450052, China; ${ }^{2}$ Department of Infectious Diseases, the First Affiliated Hospital of Zhengzhou University, Zhengzhou 450052, China; ${ }^{3}$ Gene Hospital of Henan Province; Precision Medicine Center, the First Affiliated Hospital of Zhengzhou University, Zhengzhou 450052, China; ${ }^{4}$ Department of Emergency Medicine, Shengjing Hospital of China Medical University, Shenyang 110001, China; ${ }^{5}$ Emergency Department, Chinese Academy of Medical Sciences, Peking Union Medical College Hospital, Beijing 100730, China.

\section{References}

1. Lee K, Park EK, Stoecklin-Marois M, Koivunen ME, Gee SJ, Hammock BD et al: Occupational paraquat exposure of agricultural workers in large Costa Rican farms. Int Arch Occup Environ Health 2009; 82(4):455-462.

2. Klein-Schwartz W, Smith GS: Agricultural and horticultural chemical poisonings: mortality and morbidity in the United States. Ann Emerg Med 1997; 29(2):232-238. 
3. Jones AL, Elton R, Flanagan R: Multiple logistic regression analysis of plasma paraquat concentrations as a predictor of outcome in 375 cases of paraquat poisoning. Qjm 1999; 92(10):573-578.

4. Min YG, Ahn JH, Chan YC, Ng SH, Tse ML, Lau FL et al: Prediction of prognosis in acute paraquat poisoning using severity scoring system in emergency department. Clin Toxicol (Phila) 2011; 49(9):840-845.

5. Khazraei S, Marashi SM, Sanaei-Zadeh H: Ventilator settings and outcome of respiratory failure in paraquat-induced pulmonary injury. Sci Rep 2019; 9(1):16541.

6. Wu L, Cen Y, Feng M, Zhou Y, Tang H, Liao X et al: Metformin Activates the Protective Effects of the AMPK Pathway in Acute Lung Injury Caused by Paraquat Poisoning. Oxid Med Cell Longev 2019; 2019:1709718.

7. Huang J, Xuan D, Li X, Ma L, Zhou Y, Zou H: The value of APACHE II in predicting mortality after paraquat poisoning in Chinese and Korean population: A systematic review and meta-analysis. Medicine (Baltimore) 2017; 96(30):e6838.

8. Wang WJ, Zhang LW, Feng SY, Gao J, Li Y: Sequential organ failure assessment in predicting mortality after paraquat poisoning: A meta-analysis. PLOS ONE 2018; 13(11):e0207725.

9. Sawada Y, Yamamoto I, Hirokane T, Nagai Y, Satoh Y, Ueyama M: Severity index of paraquat poisoning. Lancet 1988; 1(8598):1333.

10. Persson HE, Sjoberg GK, Haines JA, Pronczuk de Garbino J: Poisoning severity score. Grading of acute poisoning. J Toxicol Clin Toxicol 1998; 36(3):205-213.

11. Lee EY, Hwang KY, Yang JO, Hong SY: Predictors of survival after acute paraquat poisoning. Toxicol Ind Health 2002; 18(4):201-206.

12. Hong SY, Lee JS, Sun IO, Lee KY, Gil HW: Prediction of patient survival in cases of acute paraquat poisoning. PLOS ONE 2014; 9(11):e111674.

13. Hu X, Guo R, Chen X, Chen Y: Increased plasma prothrombin time is associated with poor prognosis in patients with paraquat poisoning. 2018; 32(9):e22597.

14. Wang Y, Li J, Zheng X, Jiang Z, Hu S, Wadhera RK et al: Risk Factors Associated With Major Cardiovascular Events 1 Year After Acute Myocardial Infarction. JAMA Netw Open 2018; 1(4):e181079.

15. Wei Z, Ren Z, Hu S, Gao Y, Sun R, Lv S et al: Development and validation of a simple risk model to predict major cancers for patients with nonalcoholic fatty liver disease. Cancer Med 2019.

16. Lichtman JH, Leifheit-Limson EC, Jones SB, Wang Y, Goldstein LB: Preventable readmissions within 30 days of ischemic stroke among Medicare beneficiaries. Stroke 2013; 44(12):3429-3435.

17. Hu Z, Chen S, Du J, Gu D, Wang Y, Hu S et al: An In-hospital Mortality Risk Model for Patients Undergoing Coronary Artery Bypass Grafting in China. Ann Thorac Surg 2019.

18. Wang Y: A multinomial logistic regression modeling approach for anomaly intrusion detection. Computers \& Security 2005; 24(8):662-674.

19. Schemper M, Henderson R: Predictive accuracy and explained variation in Cox regression. Biometrics 2000; 56(1):249-255.

20. O'Quigley J, Flandre P: Predictive capability of proportional hazards regression. Proc Natl Acad Sci U S A 1994; 91(6):23102314.

21. Sun B, He Y: Paraquat poisoning mechanism and its clinical treatment progress. Zhonghua Wei Zhong Bing Ji Jiu Yi Xue 2017; 29(11):1043-1046.

22. Tai W, Deng S, Wu W, Li Z, Lei W, Wang Y et al: Rapamycin attenuates the paraquat-induced pulmonary fibrosis through activating Nrf2 pathway. 2020; 235(2):1759-1768.

23. Oghabian Z, Williams J, Mohajeri M, Nakhaee S, Shojaeepour S, Amirabadizadeh A et al: Clinical Features, Treatment, Prognosis, and Mortality in Paraquat Poisonings: A Hospital-Based Study in Iran. J Res Pharm Pract 2019; 8(3):129-136.

24. Wang Y, Gao D, Geng Z, Shi Z, Wu Y, Lu S et al: Radiomics Nomogram Analyses for Differentiating Pneumonia and Acute Paraquat Lung Injury. Sci Rep 2019; 9(1):15029.

25. Zhang J, Zhao Y, Bai Y, Lv G, Wu J, Chen Y: The significance of serum uric acid level in humans with acute paraquat poisoning. Sci Rep 2015; 5:9168.

Page 12/20 
26. Gil HW, Kang MS, Yang JO, Lee EY, Hong SY: Association between plasma paraquat level and outcome of paraquat poisoning in 375 paraquat poisoning patients. Clin Toxicol (Phila) 2008; 46(6):515-518.

27. Xu S, Hu H, Jiang Z, Tang S, Zhou Y, Sheng J et al: APACHE score, Severity Index of Paraquat Poisoning, and serum lactic acid concentration in the prognosis of paraquat poisoning of Chinese Patients. Pediatr Emerg Care 2015; 31(2):117-121.

28. Jacobs S, Chang RW, Lee B: One year's experience with the APACHE II severity of disease classification system in a general intensive care unit. Anaesthesia 1987; 42(7):738-744.

29. Huang N, Lin S, Hung Y, Hung S, Chung H: Severity assessment in acute paraquat poisoning by analysis of APACHE II score. J Formos Med Assoc 2003; 102(11):782-787.

30. Gil HW, Seok SJ, Jeong DS, Yang JO, Lee EY, Hong SY: Plasma level of malondialdehyde in the cases of acute paraquat intoxication. Clin Toxicol (Phila) 2010; 48(2):149-152.

31. Liu H, Wu Q, Chu T, Mo Y, Cai S, Chen M et al: High-dose acute exposure of paraquat induces injuries of swim bladder, gastrointestinal tract and liver via neutrophil-mediated ROS in zebrafish and their relevance for human health risk assessment. Chemosphere 2018; 205:662-673.

32. Kim SJ, Gil HW, Yang JO, Lee EY, Hong SY: The clinical features of acute kidney injury in patients with acute paraquat intoxication. Nephrol Dial Transplant 2009; 24(4):1226-1232.

33. Mohamed F, Buckley NA, Jayamanne S, Pickering JW, Peake P, Palangasinghe $C$ et al: Kidney damage biomarkers detect acute kidney injury but only functional markers predict mortality after paraquat ingestion. Toxicol Lett 2015; 237(2):140150.

\section{Figures}




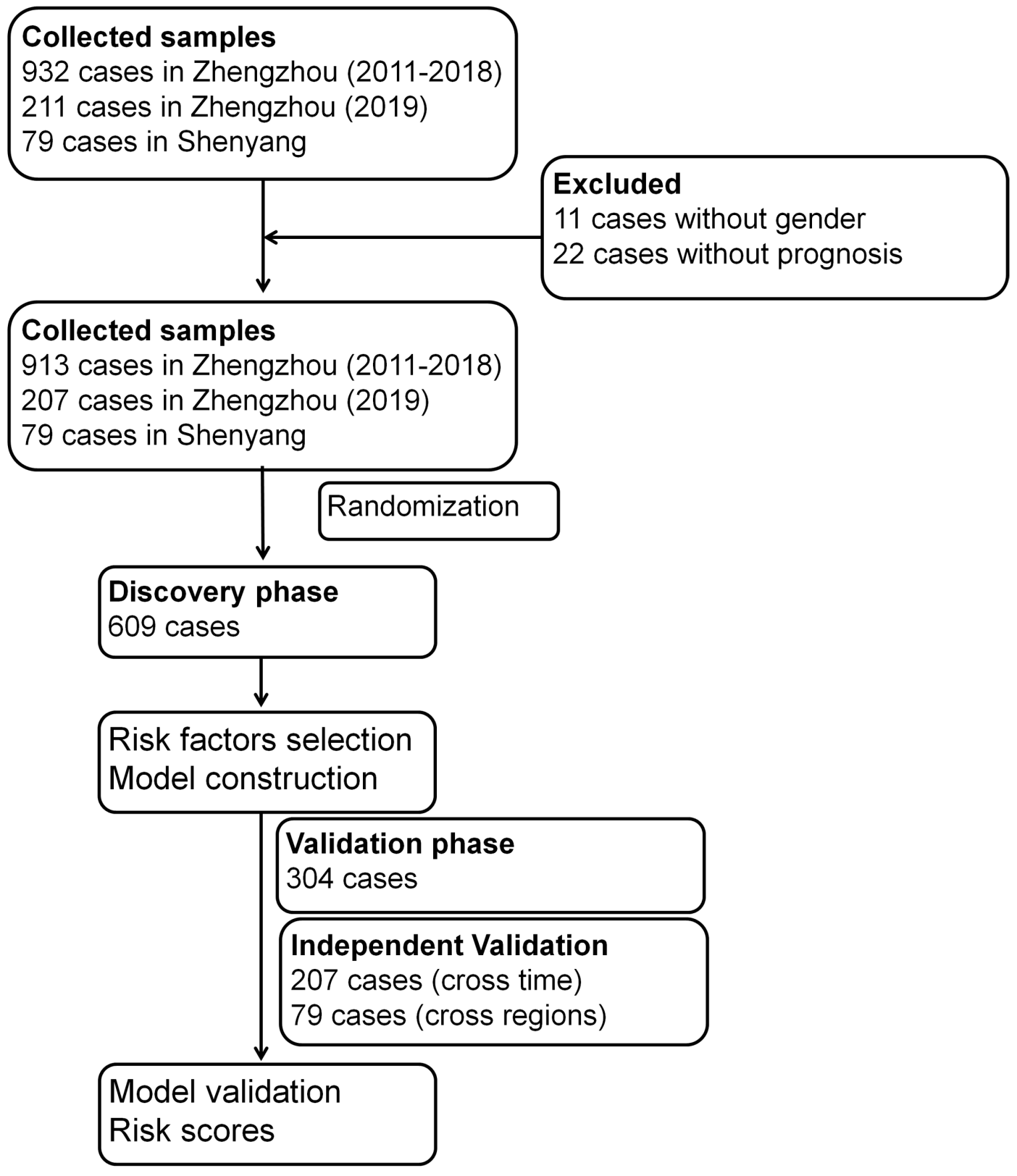

Figure 1

The design of the study. 932 samples were enrolled during the first two months in 2019. Extra 207 samples from Zhengzhou 2019 and 79 from Shenyang Shengjing hospital were collected as external validations. Patients without sex $(n=11)$ and prognosis ( $n=22)$ information were excluded. Then 913 unique patients who were randomly divided into 2 mutually exclusive samples for training (66.7\% [609 patients]) and test (33.3\% [304 patients]) group. External 207 samples from Zhengzhou 2019 were used as validation from different time and 79 from Shenyang as validation site. 


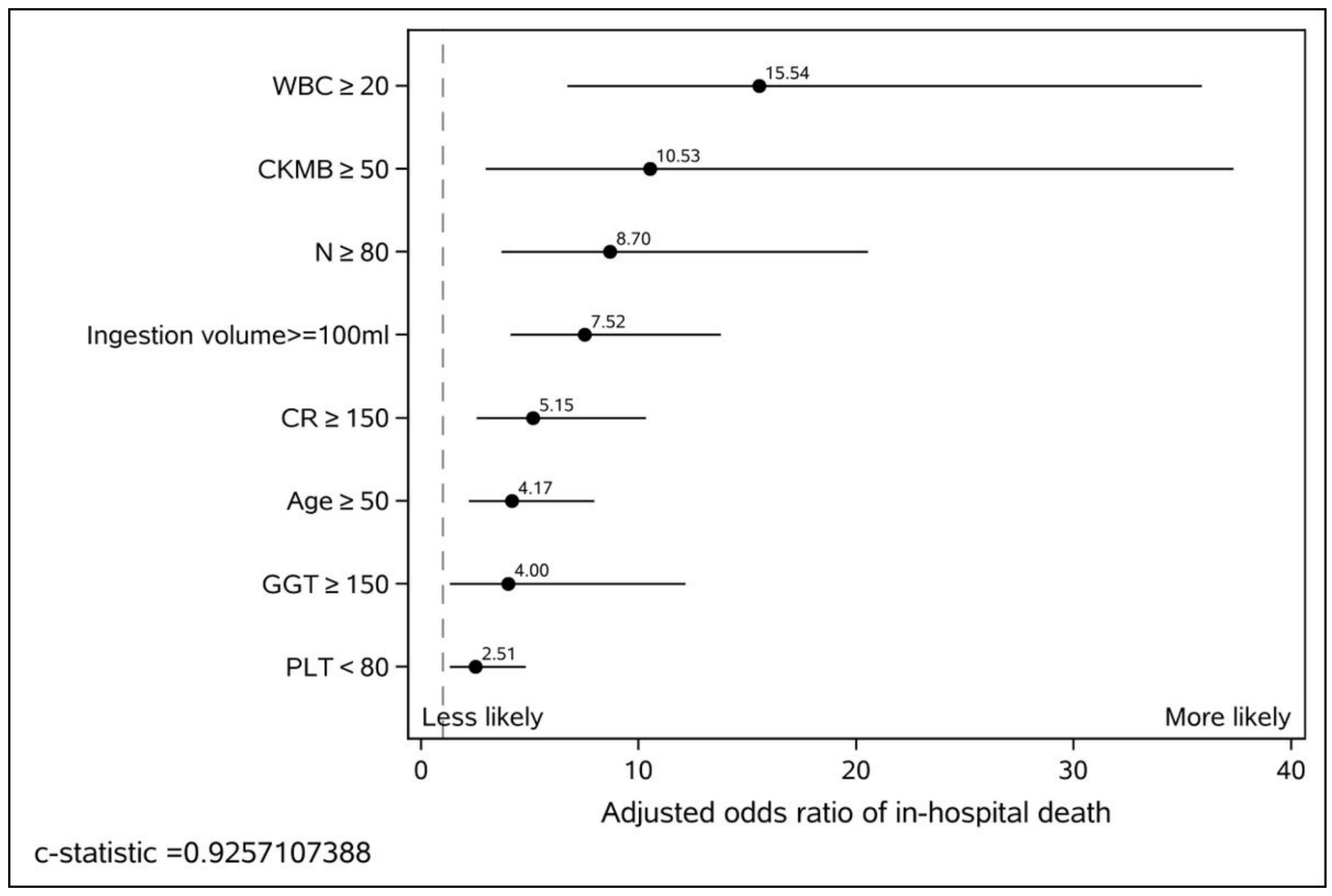

\section{Figure 2}

Risk factors associated with in-hospital death based on the training group. 8 candidate factors including age, ingestion volume, CK-MB, PLT, WBC, N, GGT and CR were identified by the MCMC simulation. The factor with a posterior probability $>0.95$ was considered significant for predicting the outcome. 


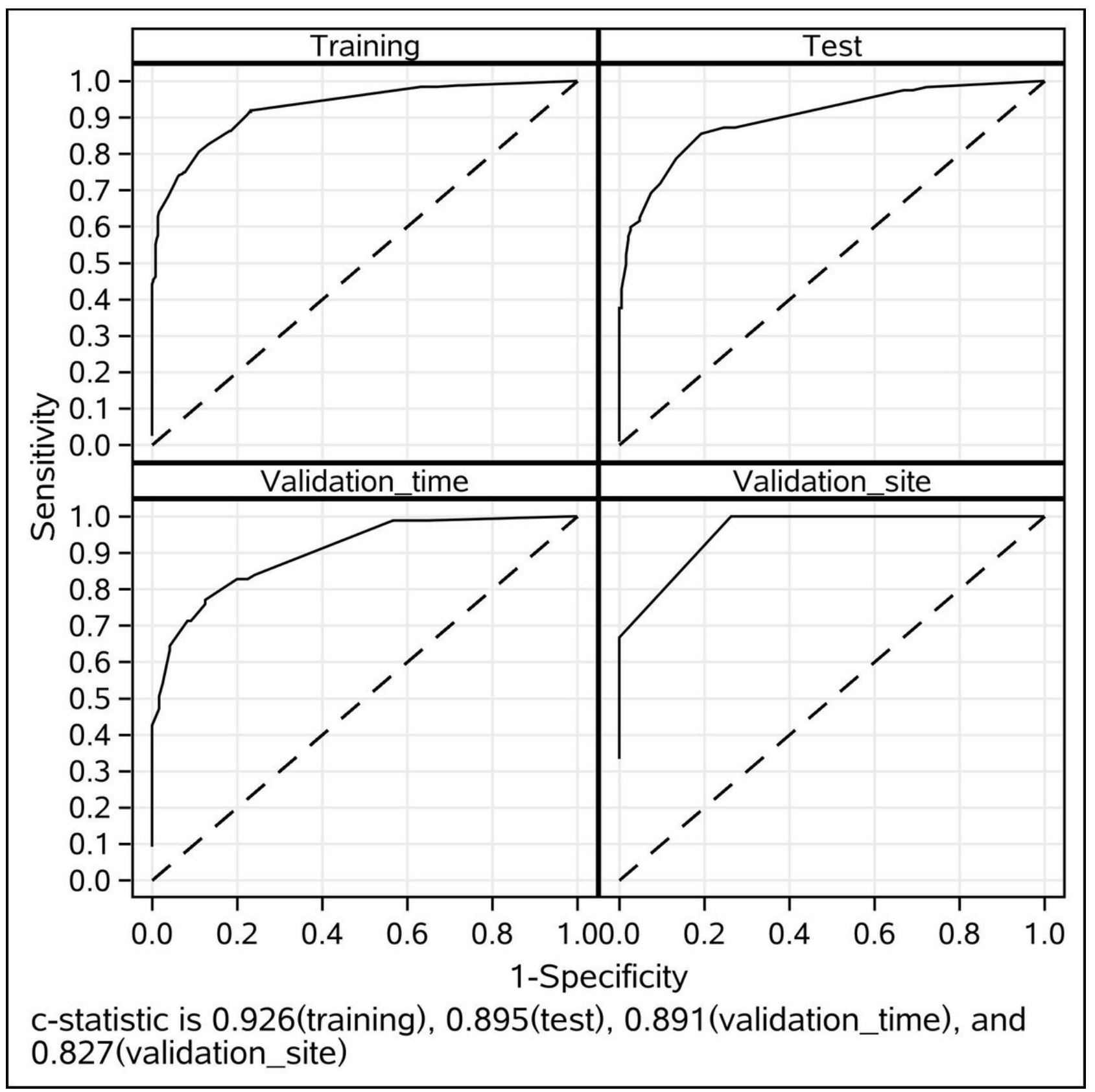

Figure 3

ROC analyses for the evaluation and validation of the risk model in the training, test, validation_time and validation_site groups. Model performance in the test, validation_time and validation_site groups was comparable to that in the training group respectively. The overall C Statistic was 0.926 (95\% Cl, 0.891-0.924), 0.895 (95\% Cl 0.855-0.928), 0.891 (95\% Cl 0.848-0.932) and 0.827 (95\% $\mathrm{Cl} 0.455-1.000)$ for the training, test, validation_time and validation_site group, respectively. 


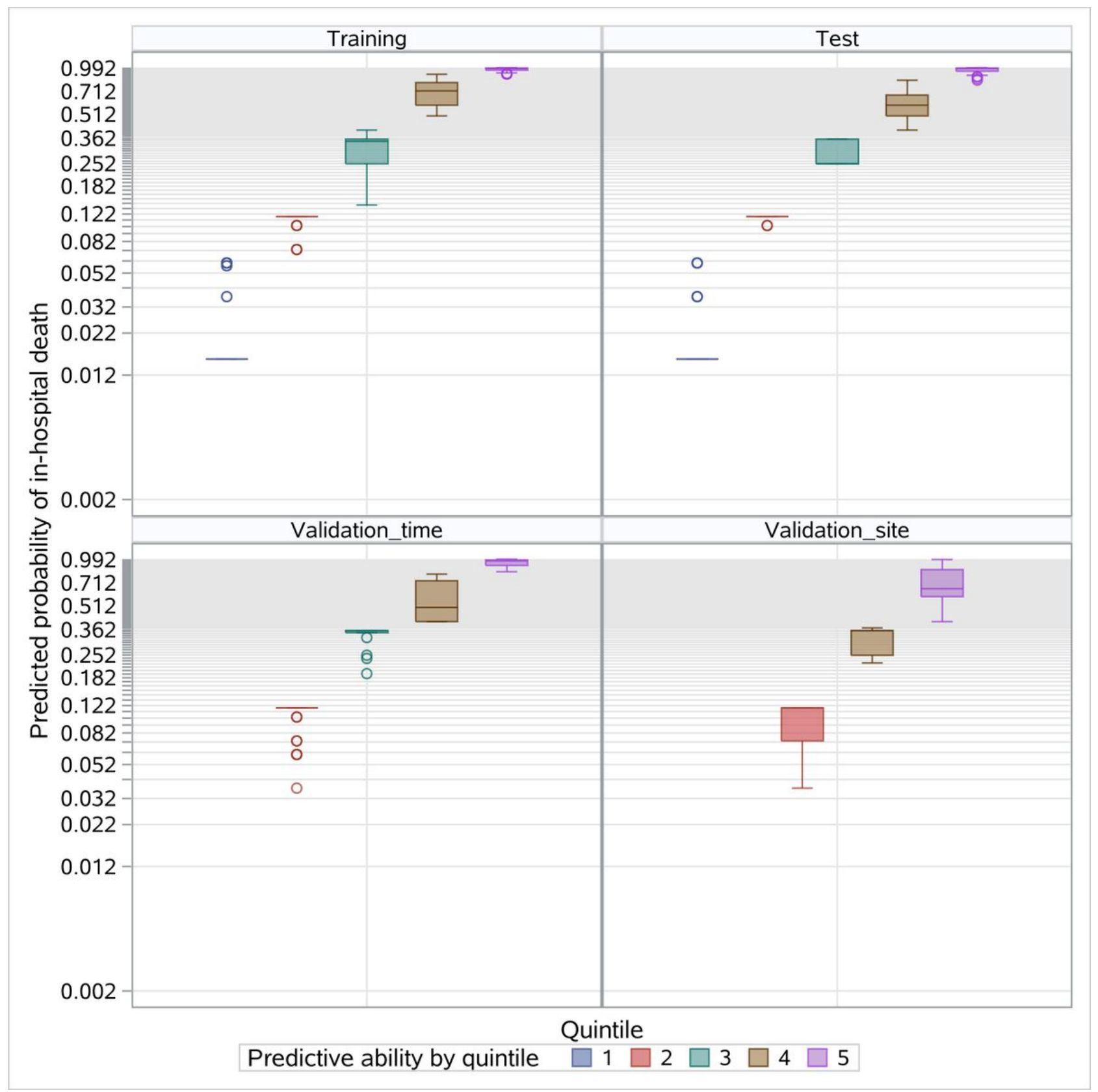

\section{Figure 4}

Probability of in-hospital death events by quintiles in the training, test, and validation_time and validation_site groups. Patients in each group were divided into 5 classes based on the combination of 8 risk factors. 


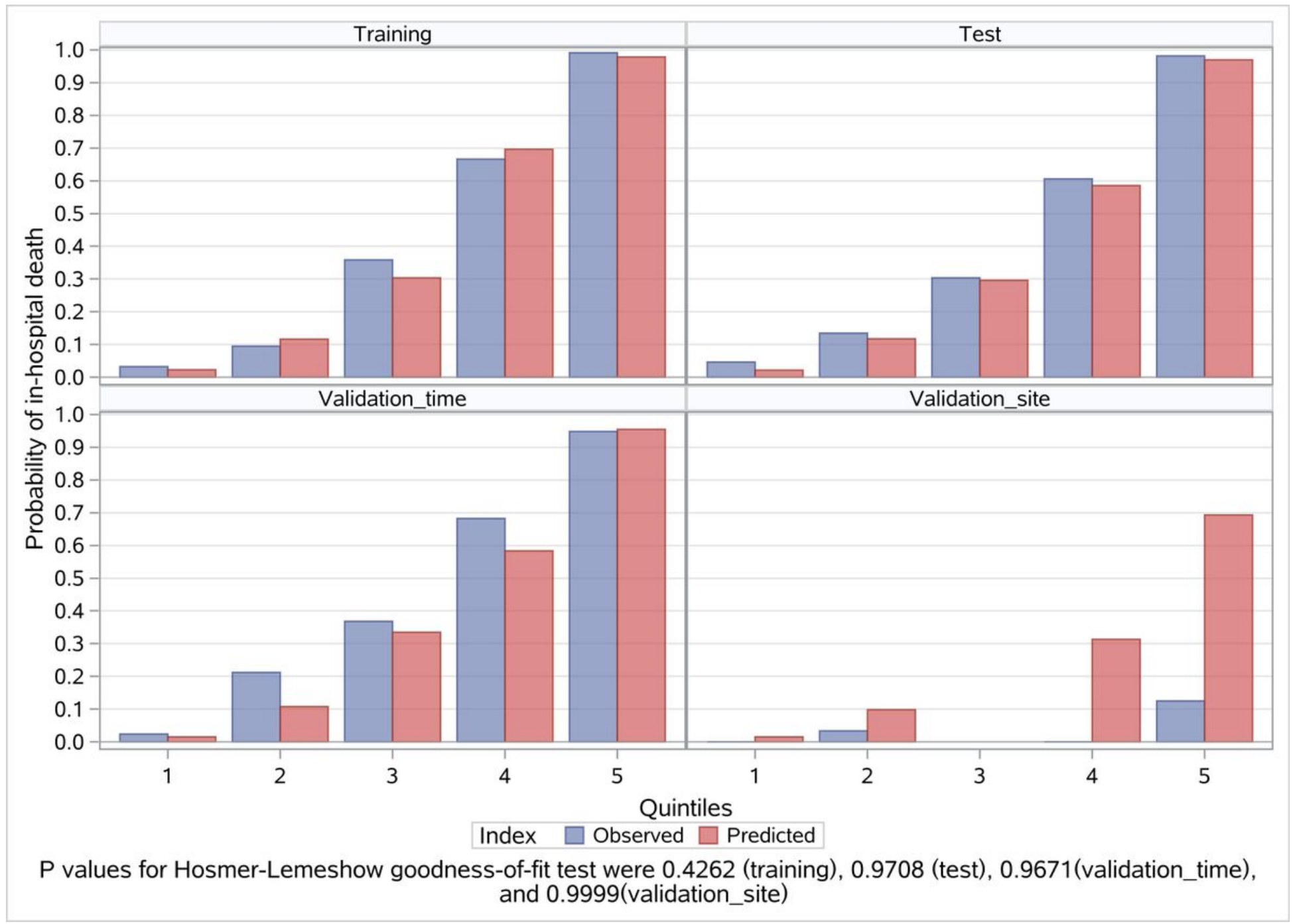

Figure 5

Observed versus predicted values by quintiles in the training, test, validation_time and validation_site groups. The Hosmer and Lemeshow's Goodness of Fit Test' p value was $0.4262,0.9708,0.9671$ and 0.9999 for the training, test, validation_time and validation_site group, respectively. 


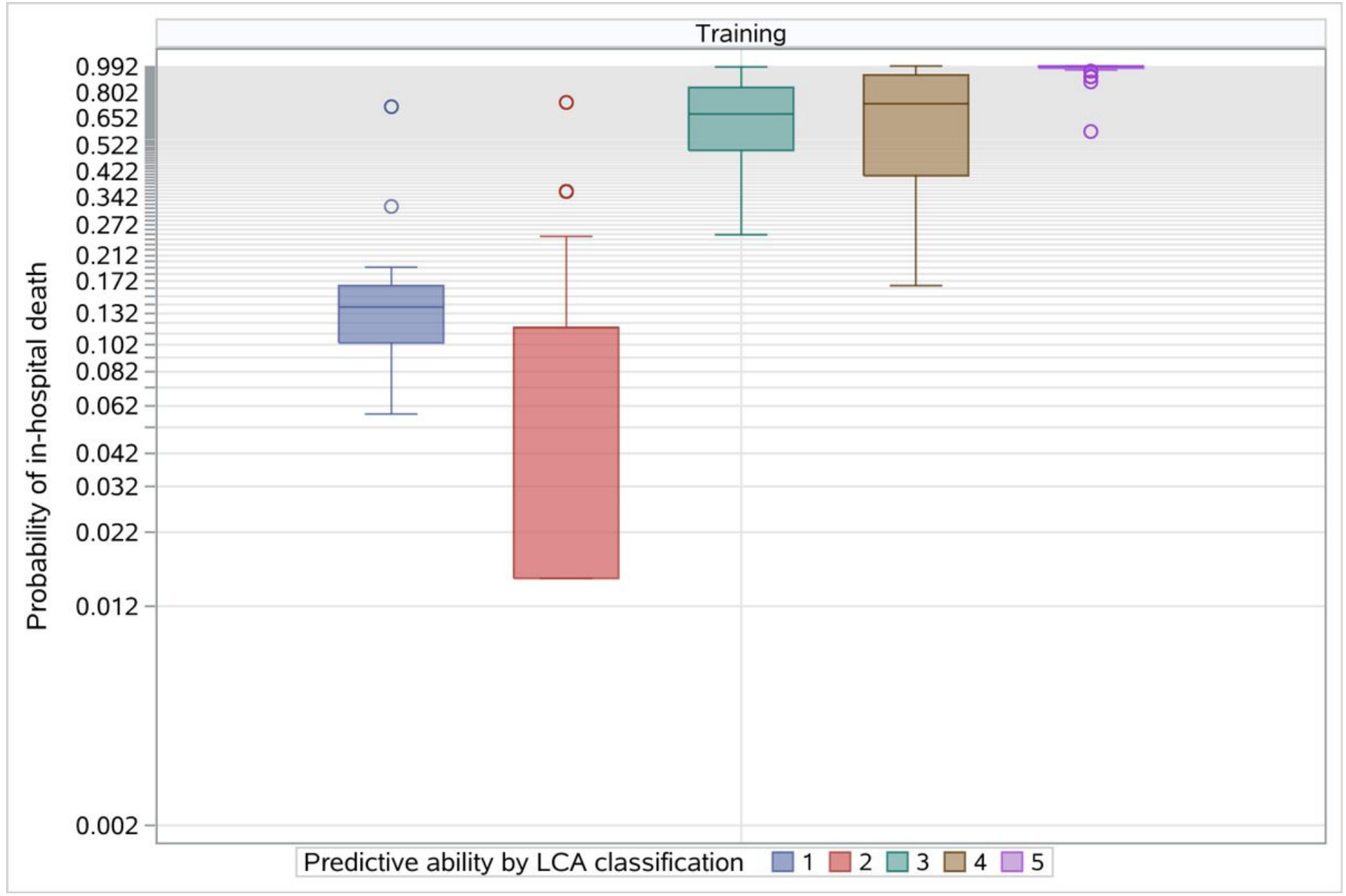

\section{Figure 6}

Risk stratification based on latent class analysis in the training group. 609 patients in the training group were divided into 5 classes based on the 8 risk factors. The mean observed outcome rate ranged from $0.0 \%$ in the lowest rating group to $99.2 \%$ in the highest rating group. 


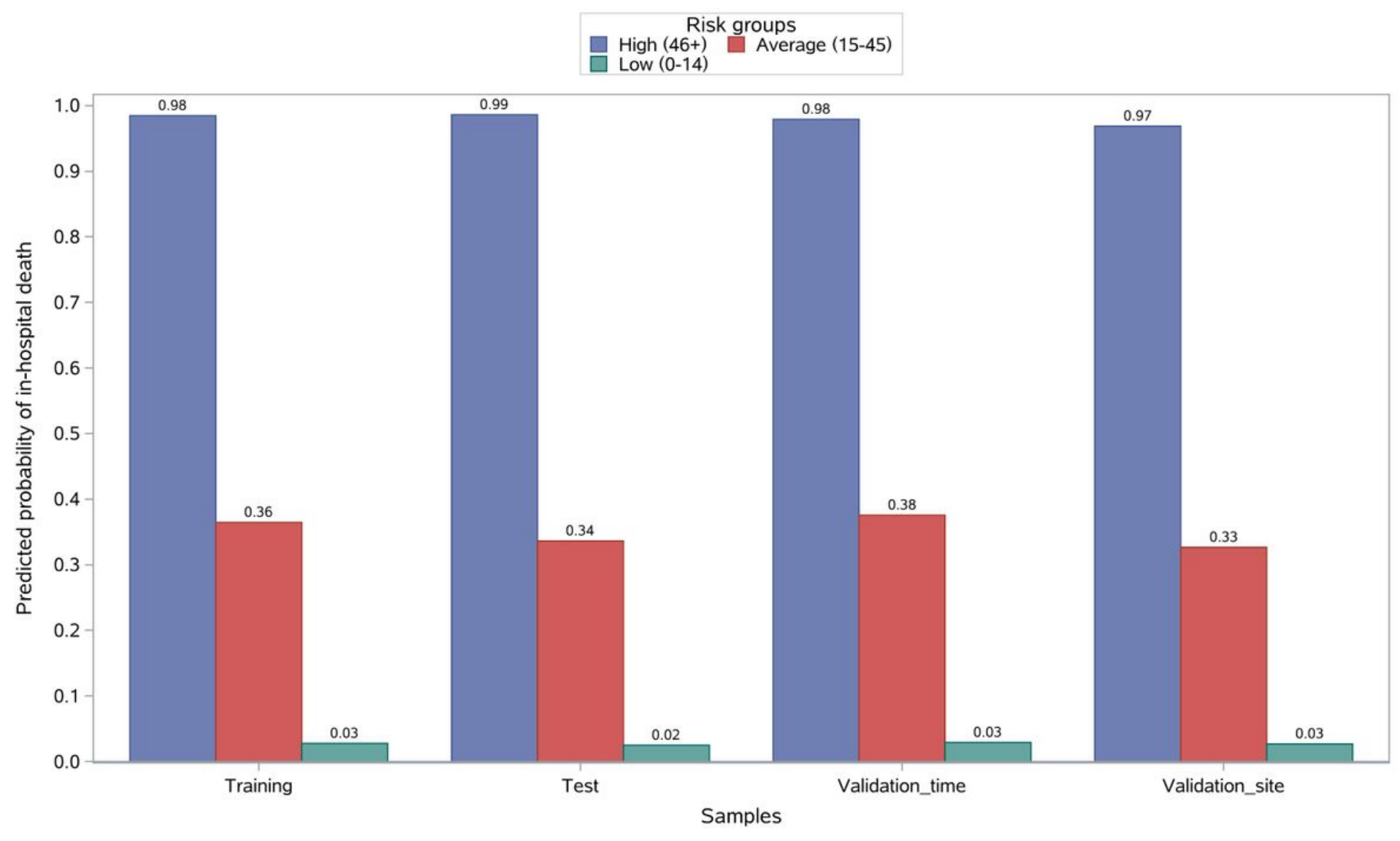

\section{Figure 7}

Risk stratification by risk scores. For the training, test, validation_time and validation_site groups, respectively, the predicted probability of in-hospital death for PQ-poisoned patients were $98 \%, 99 \%, 98 \%$ and $97 \%$ in the highest risk group (46+); $59.9 \%$, $62.2 \%, 59.4 \%$ and $60.8 \%$ in the average risk group (15-45); and $21.7 \%, 22.4 \%, 27.1 \%$ and $36.7 \%$ in the lowest risk group (0-14).

\section{Supplementary Files}

This is a list of supplementary files associated with this preprint. Click to download.

- Additionalfile.docx 